\title{
Cardioverting acute atrial fibrillation and the risk of thromboembolism: not all patients are created equal 䇺
}

\author{
Authors: Alastair J Rankin ${ }^{A}$ and Stephen H Rankin ${ }^{B}$
}

\begin{abstract}
Current guidelines support the well-established clinical practice that patients who present with atrial fibrillation (AF) of less than 48 hours duration should be considered for cardioversion, even in the absence of pre-existing anticoagulation. However, with increasing evidence that short runs of AF confer significant risk of stroke, on what evidence is this 48-hour rule based and is it time to adopt a new approach? We review existing evidence and suggest a novel approach to risk stratification in this setting. Overall, the risk of thromboembolism associated with acute cardioversion of patients with AF that is estimated to be of $<48$ hours duration is low. However, this risk varies widely depending on patient characteristics. From existing evidence, we show that using the $\mathrm{CHA}_{2} \mathrm{DS}_{2}$-VASc score may allow better selection of appropriate patients in order to prevent exposing specific patient groups to an unacceptably high risk of a potentially devastating complication.
\end{abstract}

KEYWORDS: Anticoagulants, atrial fibrillation, cardioversion, electrical cardioversion, stroke, thromboembolism

\section{Introduction}

International guidelines support the well-established clinical practice that patients who present with non-valvular atrial fibrillation (AF) of less than 48 hours duration should be considered for cardioversion, even in the absence of pre-existing anticoagulation. ${ }^{1,2}$ The risk with more prolonged periods of AF is that restoration of sinus rhythm may precipitate embolism of intra-atrial thrombus causing stroke. However, with increasing evidence that even short runs of AF confer significant risk of stroke, ${ }^{3-6}$ this review examines the evidence supporting the '48hour rule' and suggests a novel approach to risk stratification in this setting.

\section{Search strategy and selection criteria}

Data for this review were identified by searches of PubMed using combinations of the terms 'atrial fibrillation', 'cardioversion' and 'thromboembolism'. References sourced

Authors: ${ }^{\text {A }}$ clinical research fellow, Institute of Cardiovascular and Medical Sciences, University of Glasgow, UK; ${ }^{B}$ medical trainee, emergency medicine, Maroondah Hospital, Melbourne, Australia from relevant articles were also included. Additional references were identified from review of current international guidelines on the management of atrial fibrillation., ${ }^{1,2}$

\section{Is duration of AF predictive of stroke risk?}

Both paroxysmal and persistent AF increase the risk of ischaemic stroke. ${ }^{7}$ However, the risk is greater in patients with persistent arrhythmia (unadjusted annual risk of $2.1 \%$ compared to $1.5 \%)^{8,9}$ implying that the duration of AF is an important factor in determining stroke risk. Boriani et al examined data from over 10,000 patients with implanted cardiac devices to determine the critical burden (ie the duration and frequency) of paroxysmal AF that was needed to confer stroke risk. ${ }^{10}$ Overall they found that the relative risk of stroke increased by $3 \%$ for each additional hour of maximal daily AF burden. However, the threshold of AF burden that was associated with the highest relative risk for ischaemic stroke was 1 hour (hazard ratio (HR) 2.11; 95\% CI 1.22-3.64, $\mathrm{p}=0.008$. They also found that AF was commonly asymptomatic, a finding supported by other studies with one highlighting that even in patients with AF lasting $>48$ hours, $17 \%$ were asymptomatic. ${ }^{3,11}$ Therefore, if AF is commonly asymptomatic, and even paroxysms of AF lasting 1 hour confer a significant stroke risk, is duration of $\mathrm{AF}$ the best criteria for deciding whether or not to offer acute cardioversion?

\section{Risk of thromboembolism following cardioversion - where does the 48-hour rule come from?}

The '48-hour rule' was adopted into widespread clinical practice based on theoretical, rather than evidence-based, grounds. This practice was scrutinised by Weigner et al in $1997 .^{12}$ It was known that cardioversion of patients with AF of $>48$ hours duration had a $5-7 \%$ risk of stroke without preceding anticoagulation. ${ }^{12}$ However, it was also known that $14 \%$ of patients with AF of $<72$ hours duration would have atrial thrombi detectable on transoesophageal echo (TOE). ${ }^{13}$ Weigner et al prospectively examined 375 patients admitted with symptomatic AF lasting $<48$ hours in order to determine the incidence of cardioversion-related clinical thromboembolism. A total of 357 patients converted to sinus rhythm during admission, of which $66.7 \%$ did so without pharmacological or electrical attempts at rhythm control. Overall, only 3 $(0.8 \%)$ patients suffered a thromboembolic event, none of 

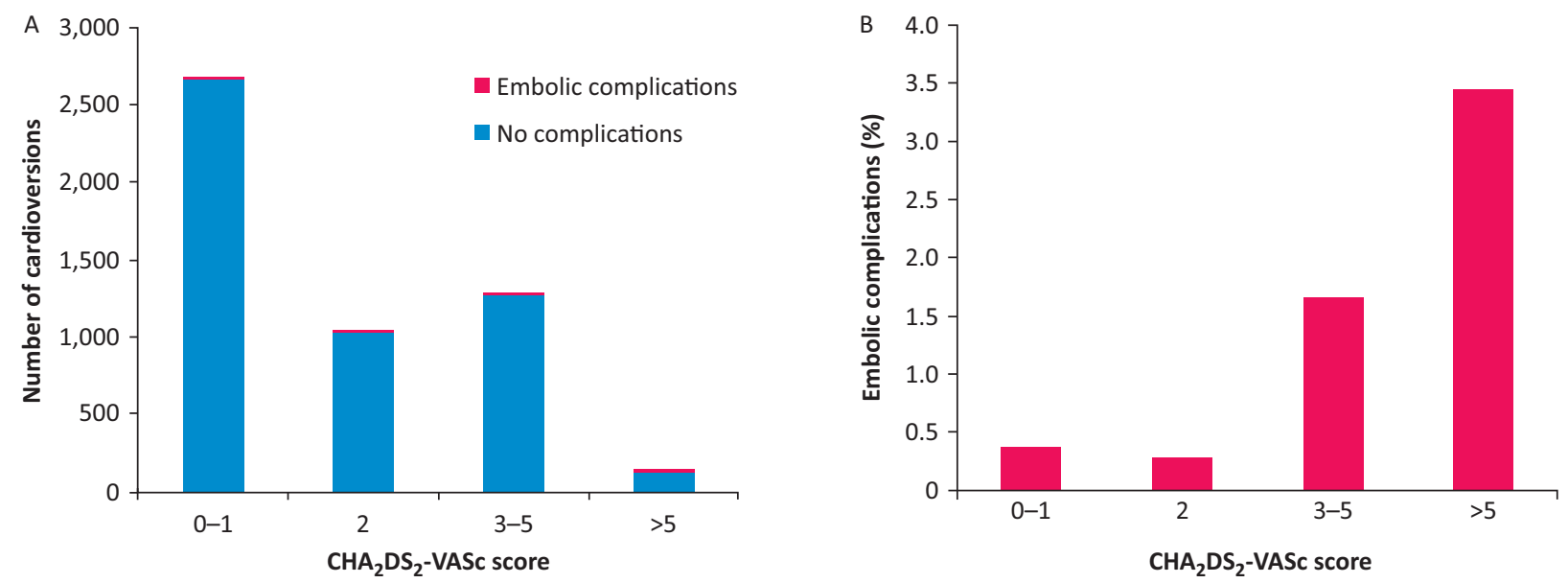

Fig 1. Embolic risk following cardioversion depends on $\mathrm{CHA}_{2} \mathrm{DS}_{2}$-VASc score. A - the absolute number of cardioversions performed without complication (blue) and with embolic complications (red) in patients with increasing $\mathrm{CHA}_{2} \mathrm{DS}_{2}-\mathrm{VASc}$ scores; $\mathrm{B}$ - the percentage of cardioversions with embolic complications in patients with increasing $\mathrm{CHA}_{2} \mathrm{DS}_{2}$-VASc scores. Data from Airaksinen et al. ${ }^{21}$

whom had been actively cardioverted. The authors concluded that early cardioversion of patients with AF of $<48$ hours duration was safe. This conclusion has been supported by several other observational studies that found the risk of thromboembolism to be negligible (ranging from $0-0.9 \%$ ) in patients who undergo cardioversion for $\mathrm{AF}$ of $<48$ hours from symptom onset. ${ }^{14-20}$ More recently, the Fin-CV study examined over 3,000 patients with AF of estimated duration $<48$ hours who were cardioverted by either pharmacological or electrical intervention. ${ }^{21}$ They confirmed that overall the risk of cardioverting patients with $\mathrm{AF}$ of $<48$ hours was low $(0.7 \%)$, with 38 thromboembolic events occurring within 30 days following cardioversion. However, this risk varied greatly depending on patient factors (Fig 1), such that risk was unacceptably high at $9.8 \%$ if a patient had heart failure and diabetes, compared with $0.2 \%$ in patients aged less than 60 years without heart failure. Interestingly, a subgroup analysis of this study found that even within 48 hours, the risk of thromboembolism varied with the duration of AF, from $0.3 \%$ if the patient was cardioverted within 12 hours compared with $1.1 \%$ beyond 12 hours (Fig 2). ${ }^{22}$

\section{Strategies to reduce the risk of thromboembolism associated with cardioversion}

Out-with the emergency setting (Box 1), acute cardioversion is a semi-elective procedure with an established alternative strategy (ie elective cardioversion after 4 weeks of reliable anticoagulation) and so only minimal levels of risk should be accepted. The authors of the Fin-CV study advocate that their results support the use of routine anticoagulation following cardioversion, especially in high-risk patients. However, with the majority of thromboembolic events occurring shortly after cardioversion (median 2 days), it could be argued that these high-risk patients should not be treated with cardioversion at all, at least not until they are established on reliable anticoagulation or a TOE has excluded intra-cardiac thrombus. ${ }^{23}$ A sustained period of anticoagulation prior to cardioversion is established practice in patients with $\mathrm{AF}>48$ hours, with some evidence to support its efficacy. ${ }^{1,2,15,17,23}$ Alternatively, TOE has been shown to be as good as 3 weeks of anticoagulation at reducing the risk of thromboembolism following cardioversion. ${ }^{23}$ TOE in this setting has the advantage of fewer haemorrhagic events, faster time to cardioversion and greater success rate at restoring sinus rhythm (presumably because of the earlier intervention) compared with patients treated with anticoagulation. ${ }^{23}$ However, these benefits must be balanced against the invasive nature of a TOE, as well as cost implications and resource constraints.

\section{A novel strategy for risk stratification: using $\mathrm{CHA}_{2} \mathrm{DS}_{2}$ - VASc}

The Fin-CV study examined patient specific risk factors and found that age, diabetes, female sex and heart failure

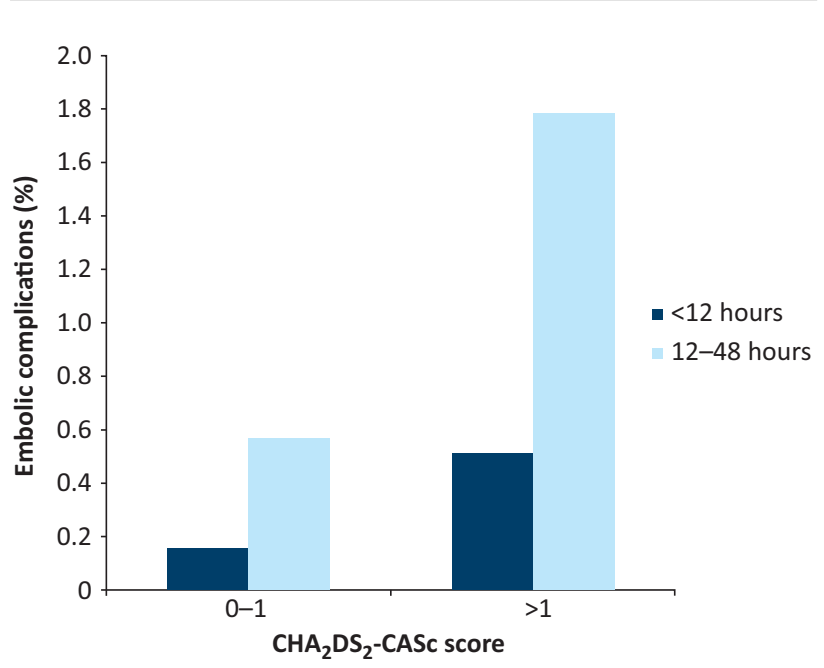

Fig 2. The effects of duration of atrial fibrillation (AF) and $\mathrm{CHA}_{2} \mathrm{DS}_{2}$ VASc score on the occurrence of embolic complications following cardioversion. Dark blue bars show embolic complications for patients cardioverted within 12 hours from onset of AF and light blue bars show those patients cardioverted 12-48 hours after onset of AF. Data from Nuotio et al. ${ }^{22}$ 
Box 1. Emergency cardioversion

Patients who present with adverse features - such as haemodynamic compromise, syncope, mycocardial ischaemia or acute pulmonary oedema - that are believed to be as a result of, or exacerbated by, the patient's atrial fibrillation should receive urgent cardioversion regardless of the duration of atrial fibrillation or anticoagulation status. ${ }^{1,2}$ All patients who receive emergency cardioversion should be anticoagulated for a minimum of 4 weeks afterwards if no contraindications. ${ }^{2}$

were all independent predictors of thromboemoblic risk. Unsurprisingly, they found the $\mathrm{CHA}_{2} \mathrm{DS}_{2}$-VASc score of thromboembolic risk, which was developed to identify long-term risk of stroke in patients with $\mathrm{AF}^{24}$ to be 'highly predictive' of thromboembolism in the acute setting. Even for patients within 48 hours of symptom onset, the risk of thromboembolism following cardioversion varied by a factor of 10 from $0.35 \%$ to $3.5 \%$ for a $\mathrm{CHA}_{2} \mathrm{DS}_{2}$-VASc score of $\leq 2$ compared with a score of $>5$ (Fig 1). Similarly, a study that examined the risk of thromboembolism with and without anticoagulation in 16,000 patients who underwent electrical cardioversion for either acute ( $<48$ hours) or chronic AF ( $>48$ hours) also found that increasing $\mathrm{CHA}_{2} \mathrm{DS}_{2}$-VASc score was associated with increasing thromboembolic risk (HR for thromboembolism within 30 days was 6.86 (95\% CI 1.55-30.37) for $\mathrm{CHA}_{2} \mathrm{DS}_{2}$-VASc of 2 compared to 0$) .^{25}$ Because all patients with a new diagnosis of AF should have their $\mathrm{CHA}_{2} \mathrm{DS}_{2}$-VASc score calculated in order to assess their long-term risk of thromboemoblism, ${ }^{1}$ it could also be used in the acute setting, in conjunction with duration of symptoms, when determining a patient's suitability for acute cardioversion. There is, however, no prospective trial data to support this recommendation. Furthermore, even using a $\mathrm{CHA}_{2} \mathrm{DS}_{2}$-VASc score cut-off of 1 would still miss $26 \%$ of the thromboembolic events in the Fin-CV study, while in the same study there were over 2,000 cardioversions performed without complication in patients with a $\mathrm{CHA}_{2} \mathrm{DS}_{2}$-VASc score $\geq 2$. This might be partly explained by the effect of AF duration on embolic risk (Fig 2) or the different weightings of individual components of $\mathrm{CHA}_{2} \mathrm{DS}_{2}$-VASc, and two patients with a score of 2 would have very different risk profiles if one had diabetes and heart failure, while the other had hypertension and vascular disease. ${ }^{21}$

\section{The role of anticoagulation peri-cardioversion}

International guidelines differ slightly with regards to the requirement of anticoagulation peri-cardioversion. ${ }^{1,2,26}$ The National Institute for Health and Care Excellence advises using $\mathrm{CHA}_{2} \mathrm{DS}_{2}$-VASc score to assess stroke risk in patients with 'continuing risk of arrhythmia recurrence after cardioversion back to sinus rhythm'. ${ }^{26}$ This advice is similar to the current European and American guidelines, which state that the decision regarding long-term anticoagulation after cardioversion should be guided by the patient's thromboembolic risk, regardless of the duration of $\mathrm{AF}$ prior to cardioversion. ${ }^{1,2}$ Where the guidelines differ is that the European guidelines state that all patients undergoing cardioversion should have a minimum of 4 weeks of anticoagulation, whereas the American guidelines advise that in patients who have had $\mathrm{AF}<48$ hours and have a low thromboembolic risk you can consider not prescribing anticoagulation at all. The rationale for anticoagulating all patients after cardioversion is to minimise the risk associated with 'atrial stunning', which promotes formation of left atrial thrombus subjecting the patient to a transient increase in their baseline risk..$^{21,27,28}$ This is supported by data from Hansen $e$ al, who found that the benefit of anticoagulation following direct current cardioversion was maintained regardless of the patient's $\mathrm{CHA}_{2} \mathrm{DS}_{2}$-VASc score. While patients with a higher $\mathrm{CHA}_{2} \mathrm{DS}_{2}-$ VASc score were more likely to develop a thromboembolic complication, the relative benefit of anticoagulation was the same irrespective of baseline risk: anticoagulation reduced the risk of thromboembolism by half even in patients with a $\mathrm{CHA}_{2} \mathrm{DS}_{2}$-VASc of 1 . However, it should be noted that this study included patients with AF $>48$ hours (in whom current guidelines would not recommend cardioversion without a period of preceding anticoagulation). Furthermore, although the relative risk reduction is maintained, the absolute risk of thromboembolism in patients with $\mathrm{CHA}_{2} \mathrm{DS}_{2}$-VASc of 1 , especially if cardioverted within 48 hours, is very low. Therefore, the absolute benefit is likely to be small and must be balanced against the small risk of 4 weeks of anticoagulation. The study of Hansen et al also found that there was no difference in the benefit of anticoagulation in patients who received it both pre- and post-procedure compared with those that received anticoagulation either pre-procedure or postprocedure only. Another study, which retrospectively analysed 567 cardioversions within 48-hours of onset, found benefit of post-procedure anticoagulation with a fivefold greater risk in those that did not receive anticoagulation after cardioversion. In this study, no thromboembolic events occurred in patients with a $\mathrm{CHA}_{2} \mathrm{DS}_{2}$-VASc $<2$ in either group. ${ }^{29}$

\section{Is acute rhythm control worth the risk?}

Overall, the risk of thromboembolism that is associated with acute rhythm control in appropriate patients is very low $(0-0.9 \%) .{ }^{14-20}$ Early rhythm control has the advantage of faster outcomes, improved symptom control and reduced hospital stay. ${ }^{30,31}$ Furthermore, the longer a patient has AF, the more difficult it is to achieve successful cardioversion to sinus rhythm. ${ }^{32}$ While some observational data ${ }^{8,9}$ supports the pursuit of rhythm control because of a reduced risk of stroke in patients with paroxysmal (as opposed to persistent) AF, this has not been replicated in clinical trials. Several studies (including the landmark AFFIRM trial) ${ }^{33}$ have shown that there is no difference in survival or thromboembolism in patients with AF who are treated with rhythm control as opposed to rate control. $^{33-38}$

\section{Is there a difference in thromboembolic risk between electrical and pharmacological cardioversion?}

There is evidence supporting the safety and efficacy of both direct current cardioversion and pharmacological cardioversion. ${ }^{16,21,39}$ Analysis of the RHYTHM-AF international registry of cardioversion found no difference in the rate of thromboembolism following electrical versus pharmacological cardioversion in 3,940 patients $(0.26 \%$ versus $0.39 \%$, respectively). ${ }^{16}$ In the Fin-CV study, there was no statistical 
comparison of electrical versus pharmacological cardioversion; however, 36 of the 38 thromboembolic complications occurred in patients treated with electrical cardioversion (accounting for a rate of $0.81 \%$ of all cardioversions or $1.65 \%$ of all patients treated with electrical cardioversion compared with rates of $0.32 \%$ and $0.67 \%$ for the corresponding figures in the pharmacological group). Electrical cardioversion has the risks associated with sedation, post-cardioversion bradycardia and skin burns. ${ }^{1}$ On the other hand, electrical cardioversion has been shown to be more effective - achieving sinus rhythm in $89 \%$ of patients compared with $69 \%$ of patients treated pharmacologically. ${ }^{16}$

\section{Conclusions}

Overall the risk of thromboembolism associated with acute cardioversion of patients with AF that is estimated to be of $<48$ hours duration is low. However, this risk varies widely depending on patient characteristics. Current guidelines expose some patients to an unacceptably high risk of thromboembolism. In our opinion, when considering and gaining consent from patients for acute cardioversion for $\mathrm{AF}$ of $<48$ hours and who are not established on anticoagulation, clinicians should be aware of a patient's individual thromboembolic risk (eg $\mathrm{CHA}_{2} \mathrm{DS}_{2}$-VASc) and if the duration of arrhythmia exceeds 12 hours from symptom onset. If both of these criteria suggest an increased risk, we would advise proceeding with caution. This strategy adds an additional safeguard to avoid exposing some patients to an unacceptably high risk of a potentially devastating complication from a non-emergency procedure. The risk of thromboembolism following cardioversion is not immediate and can persist for 30 days. Post-procedure anticoagulation reduces this risk. Anticoagulation should continue lifelong in patients with conventional thromboembolic risk factors $\left(\mathrm{CHA}_{2} \mathrm{DS}_{2}\right.$-VASc $>1)$. For low risk patients, international guidelines vary regarding the need for 4 weeks of anticoagulation postprocedure, but this should be considered and discussed with the patient.

\section{Conflicts of interest}

The authors have no conflicts of interest to declare.

\section{Author contributions}

Both authors contributed to the conception, writing and drafting of this review.

\section{References}

1 Kirchhof P, Benussi S, Kotecha D et al. 2016 ESC guidelines for the management of atrial fibrillation developed in collaboration with EACTS Eur Heart J 2016;37:2893-962.

2 January CT, Wann LS, Alpert JS et al. 2014 AHA/ACC/HRS guideline for the management of patients with atrial fibrillation: executive summary: a report of the American College of Cardiology/American Heart Association Task Force on practice guidelines and the heart rhythm society. J Am Coll Cardiol 2014;64:e1-76.

3 Glotzer T V, Ziegler PD. Cryptogenic stroke: is silent atrial fibrillation the culprit? Heart Rhythm 2015;12:234-41.
4 Sposato LA, Cipriano LE, Riccio PM et al. Very short paroxysms account for more than half of the cases of atrial fibrillation detected after stroke and TIA: a systematic review and metaanalysis. Int J Stroke 2015;10:801-7.

5 Cotter PE, Martin MPJ, Ring L et al. Incidence of atrial fibrillation detected by implantable loop recorders in unexplained stroke. Neurology 2013;80:1546-50.

6 Rojo-Martinez E, Sandin-Fuentes M, Calleja-Sanz AI et al. High performance of an implantable Holter monitor in the detection of concealed paroxysmal atrial fibrillation in patients with cryptogenic stroke and a suspected embolic mechanism. Rev Neurol 2013;57:251-7.

7 Friberg L, Hammar N, Rosenqvist M. Stroke in paroxysmal atrial fibrillation: report from the Stockholm Cohort of Atrial Fibrillation. Eur Heart J 2010;31:967-75.

8 Ganesan AN, Chew DP, Hartshorne T et al. The impact of atrial fibrillation type on the risk of thromboembolism, mortality, and bleeding: a systematic review and meta-analysis. Eur Heart $J$ 2016;37:1591-602.

9 Steinberg BA, Hellkamp AS, Lokhnygina Y et al. Higher risk of death and stroke in patients with persistent vs. paroxysmal atrial fibrillation: Results from the ROCKET-AF Trial. Eur Heart J 2015;36:288-96.

10 Boriani G, Glotzer T V, Santini M et al. Device-detected atrial fibrillation and risk for stroke: an analysis of $>10000$ patients from the SOS AF project (Stroke preventiOn Strategies based on Atrial Fibrillation information from implanted devices). Eur Heart $J$ 2014;35:508-16.

11 Israel CW, Gro G, Ehrlich JR, Li Y. Long-term risk of recurrent atrial fibrillation as documented by an implantable monitoring device implications for optimal patient care. J Am Coll Cardiol 2004;43:47-52.

12 Weigner MJ, Caulfield TA, Danias PG et al. Risk for clinical thromboembolism associated with conversion to sinus rhythm in patients with atrial fibrillation lasting less than 48 hours. Ann Intern Med 1997;126:615-20.

13 Stoddard MF, Dawkins PR, Prince CR, Ammash NM. Left atrial appendage thrombus is not uncommon in patients with acute atrial fibrillation and a recent embolic event: a transesophageal echocardiographic study. J Am Coll Cardiol 1995;25:452-9.

14 Burton JH, Vinson DR, Drummond K et al. Electrical cardioversion of emergency department patients with atrial fibrillation. Ann of Emerg Med 2004;44:20-30.

15 Gallagher MM, Hennessy BJ, Edvardsson N et al. Embolic complications of direct current cardioversion of atrial arrhythmias: association with low intensity of anticoagulation at the time of cardioversion. J Am Coll Cardiol 2002;40:926-33.

16 Crijns HJGM, Weijs B, Fairley AM et al. Contemporary real life cardioversion of atrial fibrillation: results from the multinational RHYTHM-AF study. Int J Cardiol 2014;172:588-94.

17 Gentile F, Elhendy A, Khandheria BK et al. Safety of electrical cardioversion in patients with atrial fibrillation. Mayo Clin Proc 2002;77:897-904.

18 Xavier Scheuermeyer F, Grafstein E, Stenstrom R et al. Thirty-day outcomes of emergency department patients undergoing electrical cardioversion for atrial fibrillation or flutter. Acad Emerg Med 2010;17:408-15.

19 Michael JA, Stiell IG, Agarwal S, Mandavia DP. Cardioversion of paroxysmal atrial fibrillation in the emergency department. Ann Emerg Med 1999;33:379-87.

20 Berger M, Schweitzer P. Timing of thromboembolic events after electrical cardioversion of atrial fibrillation or flutter: a retrospective analysis. Am J Cardiol 1998;82:1545-7.

21 Airaksinen KEJ, Grönberg T, Nuotio I et al. Thromboembolic complications after cardioversion of acute atrial fibrillation: the FinCV (Finnish CardioVersion) study. J Am Coll Cardiol 2013;62:1187-92. 
22 Nuotio I, Hartikainen JEK, Grönberg T et al. Time to cardioversion for acute atrial fibrillation and thromboembolic complications. JAMA 2014;312:647-9.

23 Klein AL, Grimm RA, Murray RD et al. Use of Transesophageal echocardiography to guide cardioversion in patients with atrial fibrillation. N Engl J Med 2001;344:1411-20.

24 Lip GYH, Nieuwlaat R, Pisters R et al. Refining clinical risk stratification for predicting stroke and thromboembolism in atrial fibrillation using a novel risk factor-based approach: The Euro Heart Survey on atrial fibrillation. Chest 2010;137:263-72.

25 Hansen ML, Jepsen RMHG, Olesen JB et al. Thromboembolic risk in 16274 atrial fibrillation patients undergoing direct current cardioversion with and without oral anticoagulant therapy. Europace 2014;17:18-23.

26 National Institute for Health and Care Excellence. Atrial fibrillation: management. NICE clinical guideline No 180. London: NICE, 2014.

27 Piccini JP, Fauchier L. Atrial fibrillation 3 Rhythm control in atrial fibrillation. Lancet 2016;388:829-40.

28 Grimm RA, Stewart WJ, Maloney JD et al. Impact of electrical cardioversion for atrial fibrillation on left atrial appendage function and spontaneous echo contrast: Characterization by simultaneous transesophageal echocardiography. J Am Coll Cardiol 1993;22:1359-66.

29 Garg A, Khunger M, Seicean S et al. Incidence of thromboembolic complications within 30 days of electrical cardioversion performed within 48 hours of atrial fibrillation onset. JACC Clin Electrophysiol 2016;2:487-94.

30 Thrall G, Lane D, Carroll D, Lip GYH. Quality of life in patients with atrial fibrillation: a systematic review. Am J Med 2006;119:448 e1-19.

31 Carlsson J, Miketic S, Windeler J et al. Randomized trial of ratecontrol versus rhythm-control in persistent atrial fibrillation: the strategies of treatment of atrial fibrillation (STAF) study. J Am Coll Cardiol 2003;41:1690-6.
32 Cosio FG, Aliot E, Botto GL et al. Delayed rhythm control of atrial fibrillation may be a cause of failure to prevent recurrences: Reasons for change to active antiarrhythmic treatment at the time of the first detected episode. Europace 2008;10:21-7.

33 Investigators TAFFI of RM (AFFIRM). A comparison of rate control and rhythm control in patients with atrial fibrillation. $N$ Engl J Med 2002;347:1825-33.

34 Al-Khatib SM, LaPointe NMA, Chatterjee R et al. Rate- and rhythm-control therapies in patients with atrial fibrillation: a systematic review. Ann Intern Med 2014;160:760-73.

35 Chatterjee S, Sardar P, Lichstein E et al. Pharmacologic rate versus rhythm-control strategies in atrial fibrillation: an updated comprehensive review and meta-analysis. Pacing Clin Electrophysiol 2013;36:122-33.

36 de Denus S, Sanoski CA, Carlsson J et al. Rate vs rhythm control in patients with atrial fibrillation: a meta-analysis. Arch Intern Med 2005; 165:258-62.

37 Roy D, Talajic M, Nattel S et al. Rhythm control versus rate control for atrial fibrillation and heart failure. $N$ Engl J Med 2008;358:2667-77.

38 Van Gelder IC, Hagens VE, Bosker HA et al. A comparison of rate control and rhythm control in patients with recurrent persistent atrial fibrillation. N Engl J Med 2002;347:1834-40.

39 Kriz R, Freynhofer MK, Weiss TW et al. Safety and efficacy of pharmacological cardioversion of recent-onset atrial fibrillation: a single-center experience. Am J Emerg Med 2016;34:1486-90.

Address for correspondence: Dr Alastair Rankin, Institute of Cardiovascular and Medical Sciences, University of Glasgow, Glasgow G12 8TA, UK.

Email: alastair.rankin@nhs.net

\section{NGC

New to systematic reviewing, or need to improve your critical appraisal skills?

Our intensive 1-day courses will introduce you to the key principles of critical appraisal and systematic reviewing, and equip you with the basic skills to put your knowledge into practice.

$>$ Introduction to critical appraisal

> Systematic reviews and meta-analysis in action

Discount available if both courses are booked together.

\section{'Informative and practical'} Dr Rajeswari Ramaraj

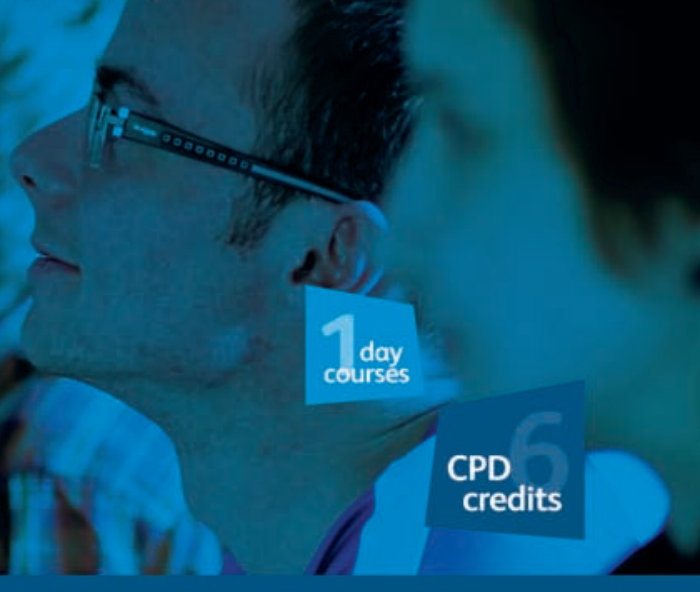

For course dates and more information visit: www.ngc.ac.uk/training-courses 